\title{
Transforming growth factor- $\beta 1$-induced epithelial-mesenchymal transition in human esophageal squamous cell carcinoma via the PTEN/PI3K signaling pathway
}

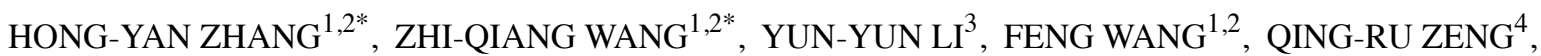 \\ YUAN GAO ${ }^{1,2}$, XIAO-YAN XUAN ${ }^{5}$ and SHAN-SHAN LI ${ }^{1,2}$ \\ ${ }^{1}$ Department of Pathology, The First Affiliated Hospital of Zhengzhou University, Zhengzhou, \\ Henan 450052; ${ }^{2}$ Department of Pathology, Basic Medical College of Zhengzhou University, Zhengzhou, \\ Henan 450001; ${ }^{3}$ Department of Stomatology, The First Affiliated Hospital of Zhengzhou University, \\ Zhengzhou, Henan 450052; ${ }^{4}$ Department of Supersonics, The Third Affiliated Hospital of Zhengzhou University, \\ Zhengzhou, Henan 450052; ${ }^{5}$ Department of Microbiology and Immunology, Basic Medical College of \\ Zhengzhou University, Zhengzhou, Henan 450001, P.R. China
}

Received April 30, 2014; Accepted August 6, 2014

DOI: $10.3892 / o r .2014 .3453$

\begin{abstract}
Epithelial-mesenchymal transition (EMT) is a crucial step for the invasive and metastatic properties of malignant tumor cells during tumor progression. Numerous signaling pathways are involved in the process of EMT in cancer, such as the EMT-inducing signal transforming growth factor (TGF)- $\beta$ and the recently demonstrated PTEN/PI3K signaling pathway. To date, no data have been reported concerning the influence of PTEN/PI3K signaling pathway on EMT in human esophageal squamous cell carcinoma (ESCC) and how TGF- $\beta 1$ and PTEN/PI3K act through multiple interconnected signaling pathways to trigger events associated with EMT and tumor progression. Our data showed that the PTEN/PI3K pathway was active in human ESCC tissues in vivo, particularly in ESCC with decreased E-cadherin and increased vimentin protein expression, poor differentiation, deep invasion and lymph node metastasis, which are responsible for EMT and tumor progression. In addition, in the human ESCC cell line (EC-1) in vitro, TGF- $\beta 1$ treatment markedly induced EMT, including morphological alterations, a decrease of E-cadherin and an increase of vimentin levels and enhanced mobility and invasiveness. Furthermore, the PTEN/PI3K pathway was also activated in the process of TGF- $\beta 1$-induced EMT in EC-1 cells in vitro, whereas inhibition of the PTEN/PI3K pathway by using pcDNA3.1 PTEN partially blocked TGF- $\beta 1$-induced EMT and reduced mobility and invasiveness. These studies
\end{abstract}

Correspondence to: Professor Shan-Shan Li, Department of Pathology, The First Affiliated Hospital of Zhengzhou University, 40 Daxue Road, Zhengzhou, Henan 450052, P.R. China

E-mail: 1sspath@163.com

*Contributed equally

Key words: epithelial-mesenchymal transition, transforming growth factor- $\beta 1$, PTEN, PI3K, esophageal squamous cell carcinoma suggest that TGF- $\beta 1$ and the PTEN/PI3K signaling pathway contribute to EMT and the PTEN/PI3K signaling pathway is a key regulator of TGF- $\beta 1$-induced EMT in ESCC. Disruption of the PTEN/PI3K pathway involved in TGF- $\beta 1$-induced EMT may provide possible routes for therapeutic intervention to ESCC.

\section{Introduction}

Esophageal carcinoma is one of the most common malignant tumors in the world. It has a high incidence rate in China, especially in Henan Province, with a poor prognosis (1-3). In spite of significant advancement in early diagnosis, surgical intervention as well as local and systemic adjuvant therapies, the majority of cancer deaths are attributable to tumor invasion and metastasis that are resistant to available therapies $(4,5)$. Notably, it was found that malignant tumor cells increased their cell invasion and motility in a manner similar to the epithelial-to-mesenchymal transition (EMT) (6).

EMT is a highly conserved process required for embryonic development, tissue remodeling and wound repair, and is also implicated in the progression of primary tumors through invasion and metastasis (7-10). During the process of EMT, the epithelial cells lose their epithelial characteristics, including their polarity and specialized cell-cell contacts, are converted to a mesenchymal phenotype and further acquire a migratory behavior, allowing them to move away from their epithelial cell community and to integrate into surrounding tissue, even at remote locations (11). Therefore, EMT is thought to play a fundamental role in the invasion and metastasis of cancer cells (12). A hallmark of EMT is the downregulation of epithelial markers including E-cadherin and $\beta$-catenin and the upregulation of markers related to the mesenchymal phenotype or fibrosis including vimentin, collagens and fibronectin $(11,13)$.

Numerous signaling pathways are involved in the process of EMT in human cancer (14). Transforming growth factor 
(TGF)- $\beta$, as a common and potent EMT-inducing signal $(11,15)$, can initiate cancer cells to undergo the EMT process and thus develop an aggressive and invasive phenotype. It has been shown, for example, that TGF- $\beta$ can induce EMT in various types of human cancer (16-18). However, the complex molecular mechanisms underlying this TGF- $\beta$-induced EMT process are not yet fully understood. The tumor suppressor gene PTEN, a negative regulator of oncogenic PTEN/PI3K signaling pathway, can directly antagonize PI3K signal, thereby negatively regulating aggressive tumor behavior $(19,20)$. Recent reports demonstrated that the PTEN/PI3K signaling pathway contributes to EMT in cancer $(21,22)$. Since TGF- $\beta$ and the PTEN/PI3K signaling pathway fulfill overlapping roles in tumor progression, the study of the possible role of PTEN/PI3K in TGF- $\beta$-induced EMT is warranted to shed light for future research. To date, no data have been reported concerning the influence of TGF- $\beta$ on the PTEN/PI3K signaling pathway in the ESCC cells and the role of this interaction on crucial characteristics of tumor cells, such as invasion and migration.

In the present study, we provided insight into how TGF- $\beta 1$ and PTEN/PI3K act through multiple interconnected signaling pathways to trigger events associated with EMT in esophageal squamous cell carcinoma (ESCC). We examined the expression profile of PTEN/PI3K signal in patients with ESCC and compared PTEN/PI3K expression with E-cadherin/vimentin expression and clinicopathological parameters (such as invasion depth and lymph node metastasis), which are all associated with EMT and tumor progression. Furthermore, we investigated the effect of TGF- $\beta 1$ and PTEN/PI3K on phenotypic and functional characteristics consistent with EMT in ESCC cells and whether a crosstalk between TGF- $\beta 1$ and PTEN/PI3K signaling pathway is required to mediate this effect.

\section{Materials and methods}

Tissue samples. The present study included formalin-fixed and paraffin-embedded (FFPE) tissue samples of 95 patients with histopathologically confirmed ESCC from The First Affiliated Hospital of Zhengzhou University, Henan, China. None of the patients had received neoadjuvant radio-/chemotherapy. Clinical samples used in this study were approved by the Committee for Ethical Review of Research in our hospital. Clinical information on the samples is summarized in Table I.

Immunohistochemistry (IHC). Sections of the 95 FFPE tissue specimens were processed for PTEN (Zhongshan, Beijing, China; 1:100), PI3K (Zhongshan; 1:100), E-cadherin (Zhongshan; 1:150) and vimentin (Zhongshan; 1:100) staining according to previously established protocols (23). Briefly, $5 \mu \mathrm{m}$ serial sections were deparaffinized, subsequently rehydrated in gradients of ethanol, subjected to antigen retrieval and incubated with primary antibodies overnight. Signal visualization was developed by the avidin-biotin peroxidase method using DAB, following immunohistochemical staining analysis performed by two independent pathologists.

Evaluation of IHC staining. We assessed immunohistochemical staining only in normal epithelial and cancer cells. PTEN, PI3K and vimentin positive signals all showed brown-yellow granules in the cytoplasm, whereas E-cadherin showed it in the membrane or cytoplasm. Under a microscope at $\mathrm{x} 400$ magnification, five fields of vision (FOVs) were randomly selected (for each FOV, there were no fewer than 200 cells) and the results were interpreted in accordance with the percentage of cells and depth of stain. (i) Scored in accordance with the percentage of positive cells in like-kind cells: $1,<30 \%$; $2,30-70 \% ; 3,>70 \%$. (ii) Scored in accordance with depth of stain: 0 , no cell coloration; 1 , light yellow; 2 , brown; 3 , tan. The product of (i) and (ii) was used as the total multiplied score, where $0-1$ indicates a negative score (-) and $\geq 2$ a positive score (+) (23).

Cell line. The human ESCC cell line EC-1, a gift from Professor Shihua Cao (the University of Hong Kong), was cultured in RPMI-1640 medium (Gibco, USA) supplemented with $10 \%$ fetal bovine serum (FBS), $100 \mu \mathrm{g} / \mathrm{ml}$ streptomycin and $100 \mathrm{U} / \mathrm{ml}$ penicillin at $37^{\circ} \mathrm{C}$ in a humidified incubator containing $5 \% \mathrm{CO}_{2}$. The experiment cells were in logarithmic phase.

pcDNA3.1-PTEN plasmid construction and stable transfection. Human PTEN cDNA was subcloned into pcDNA3.1 (Invitrogen, USA) vector with primers as follows: forward, 5'-AAGCTTATGACAGCCATCATCAAAGAGAT-3' (underlined, HindIII) and reverse, 5'-GGATCCGGAATAAAACGG GAAAGTGCC-3' (underlined, BamHI). pcDNA3.1-PTEN and control empty pcDNA3.1 vector were transfected into EC-1 cells using Lipofectamine ${ }^{\mathrm{TM}} 2000$ (Invitrogen) according to the manufacturer's protocol, respectively. G418 was used to select stable expression clones.

Cell treatment. To evaluate the effect of TGF- $\beta 1$ on EMT, EC- 1 cells were treated with $5 \mathrm{ng} / \mathrm{ml}$ of TGF- $\beta 1$ (PeproTech, Rocky Hill, NJ, USA) for different time intervals $(0,24$ and $48 \mathrm{~h}$ ). To analyze the crosstalk of TGF- $\beta 1$ and PTEN/PI3K pathway during the process of EMT, EC-1 cells were stably transfected with pcDNA3.1-PTEN and subsequently treated with $5 \mathrm{ng} / \mathrm{ml}$ of TGF- $\beta 1$ for $48 \mathrm{~h}$. After different treatment, cells were observed and photographed for the morphological alterations under a reversed light microscope and analyzed for mRNA and protein levels of E-cadherin, vimentin, PTEN and $\mathrm{PI} 3 \mathrm{~K}$, cell motility and invasiveness as described below.

$R N A$ preparation and quantitative real-time $P C R$. Total cellular RNA was extracted from EC-1 cells with different treatment by the TRIzol reagent (Invitrogen) according to the manufacturer's protocols. Total RNA $(2 \mu \mathrm{g})$ was used to generate the first strand of DNA using the TIANScript First Strand cDNA Synthesis kit (Tiangen, China). Quantitative real-time PCR (qPCR) was carried out with primers specific for PTEN (forward, 5'-ATACCAGGACCAGAGGAAACC-3' and reverse, 5'-TTGTCATTATCCGCACGCTC-3'); PI3K (forward, 5'-TGTAGTGGTGGACGGCGAAGTA-3' and reverse, 5'-GGGAGGTGTGTTGGTAATGTAGCA-3'); E-cadherin (forward, 5'-TGATTCTGCTGCTCTTGCTG and reverse, 5'-CAAAGTCCTGGTCCTCTTCTCC-3'); vimentin (forward, 5'-AATGACCGCTTCGCCAACTA-3' and reverse, 5'-GCTCCTGGATTTCCTCTTCG-3') and $\beta$-actin (forward, 5'-CGGGAAATCGTGCGTGAC-3' and reverse 5'-TGGAA 
Table I. Expression of E-cadherin, vimentin, PTEN and PI3K proteins in ESCC tissues and their correlation with clinicopathological parameters.

\begin{tabular}{|c|c|c|c|c|c|c|c|c|c|c|c|c|c|}
\hline & \multirow[b]{2}{*}{$\mathrm{n}$} & \multicolumn{3}{|c|}{ E-cadherin } & \multicolumn{3}{|c|}{ Vimentin } & \multicolumn{3}{|c|}{ PTEN } & \multicolumn{3}{|c|}{ PI3K } \\
\hline & & - & + & P-value & - & + & P-value & - & + & P-value & - & + & P-value \\
\hline Normal mucosa & 95 & 22 & 73 & 0.000 & 67 & 28 & 0.000 & 10 & 85 & 0.000 & 50 & 45 & 0.001 \\
\hline ESCC & 95 & 57 & 38 & & 39 & 56 & & 48 & 47 & & 28 & 67 & \\
\hline \multicolumn{14}{|l|}{ Gender } \\
\hline Male & 55 & 31 & 24 & 0.396 & 23 & 32 & 0.859 & 30 & 25 & 0.358 & 16 & 39 & 0.924 \\
\hline Female & 40 & 26 & 14 & & 16 & 24 & & 18 & 22 & & 12 & 28 & \\
\hline \multicolumn{14}{|l|}{ Age (years) } \\
\hline$<60$ & 42 & 28 & 14 & 0.238 & 20 & 22 & 0.247 & 25 & 17 & 0.118 & 13 & 29 & 0.778 \\
\hline$\geq 60$ & 53 & 29 & 24 & & 19 & 34 & & 23 & 30 & & 15 & 38 & \\
\hline \multicolumn{14}{|l|}{ Histological grade } \\
\hline I & 21 & 7 & 14 & 0.017 & 8 & 13 & 0.717 & 4 & 17 & 0.000 & 9 & 12 & 0.117 \\
\hline II & 44 & 29 & 15 & & 20 & 24 & & 19 & 25 & & 14 & 30 & \\
\hline III & 30 & 21 & 9 & & 11 & 19 & & 25 & 5 & & 5 & 25 & \\
\hline \multicolumn{14}{|l|}{ Invasion depth } \\
\hline Superficial muscularis & 24 & 6 & 18 & 0.000 & 18 & 6 & 0.000 & 6 & 18 & 0.004 & 15 & 9 & 0.000 \\
\hline Deep muscularis & 71 & 51 & 20 & & 21 & 50 & & 42 & 29 & & 13 & 58 & \\
\hline \multicolumn{14}{|l|}{ Lymph node metastasis } \\
\hline Yes & 30 & 23 & 7 & 0.024 & 10 & 20 & 0.299 & 27 & 3 & 0.000 & 4 & 26 & 0.019 \\
\hline No & 65 & 34 & 31 & & 29 & 36 & & 21 & 44 & & 24 & 41 & \\
\hline
\end{tabular}

GGTGGACAGCGAGG-3'). All reactions were performed in triplicate on a 7300 real-time PCR detection system (Applied Biosystems, Foster City, CA, USA), using SYBR Green (Applied Biosystems) fluorescent dye and normalized to $\beta$-actin mRNA levels.

Western blot analysis. EC-1 cells with different treatment were harvested and lysed using the entire protein extraction reagent (KeyGen, Nanjing, China) according to the manufacturer's protocols. Thirty micrograms of protein for each sample were electrophoresed through a $10 \%$ SDS-PAGE gel and then electrotransferred to nitrocellulose membranes (Yili, China) by a semi-dry transfer apparatus. The membranes were incubated at $4^{\circ} \mathrm{C}$ overnight with the following primary antibodies: PTEN (Zhongshan; 1:500), PI3K (Zhongshan; 1:500), E-cadherin (Zhongshan; 1:800), vimentin (Zhongshan; 1:1000) and $\beta$-actin (Zhongshan; 1: 500). $\beta$-actin was measured to control for equal loading. The experiments were performed in triplicate.

Cell invasion assay. Tumor cell invasive ability was analyzed using an invasion chamber (Oilin, China) with Matrigel-coated polycarbonate membrane ( $8 \mu \mathrm{m}$ pore size) in 24 -well plates. EC-1 cells with different treatment were seeded into the upper compartment of the invasion chamber containing serumfree medium and RPMI-1640 with 10\% FBS was added in the lower chamber as a chemoattractant. After incubation at $37^{\circ} \mathrm{C}$ in $5 \% \mathrm{CO}_{2}$ for $24 \mathrm{~h}$, cells that invaded through the Matrigel and adhered to the lower surface were fixed in $95 \%$ alcohol, stained with HE and counted under a reversed light microscope. Data represent the mean $( \pm \mathrm{SD})$ of the number of cells counted in five random FOVs at x400 magnification in each of 3 independent experiments.

Scratch assay. Tumor cell migratory ability was measured by the scratch assay. EC-1 cells transfected with or without pcDNA3.1-PTEN were planted in 6-well plates and grown to $100 \%$ confluency, respectively. A wound was then introduced to each well by scratching the monolayer cells with a sterile $200 \mu 1$ pipette tip. The cultures were washed with PBS to remove detached cells. Subsequently, cells were allowed to grow with or without treatment of $5 \mathrm{ng} / \mathrm{ml}$ of TGF- $\beta 1$ for an additional $48 \mathrm{~h}$. At different time intervals $(0,24$ and $48 \mathrm{~h})$, cell migration was photographed at a $\times 200$ magnification under a reversed light microscope, quantitated by measuring the width of the wounds at least five representative fields and expressed as 1 minus the average percent of wound closure by comparing with that at the zero time. Experiments were performed with at least 6 replicates for each condition.

Statistical analysis. Data analyses were carried out with the Chi-square test or t-test using SPSS version 13.0. In all statistical analyses, P-value $<0.05$ was considered to indicate a statistically significant difference and all P-values were two-sided.

\section{Results}

A hallmark of EMT in ESCC tissues: downregulation of epithelial marker E-cadherin and upregulation of 
Table II. Correlation of E-cadherin with vimentin protein expression in ESCC tissues.

\begin{tabular}{lcccc}
\hline & \multicolumn{4}{c}{ Vimentin } \\
\cline { 2 - 5 } E-cadherin & + & - & $\mathrm{r}$ & P-value \\
\hline+ & 10 & 28 & -0.542 & 0.000 \\
- & 46 & 11 & & \\
\hline
\end{tabular}

Table III. Correlation of PTEN with PI3K protein expression in ESCC tissues.

\begin{tabular}{lcccc}
\hline & \multicolumn{5}{c}{ PI3K } \\
\cline { 2 - 5 } PTEN & + & - & r & P-value \\
\hline+ & 20 & 27 & -0.607 & 0.000 \\
- & 47 & 1 & & \\
\hline
\end{tabular}

mesenchymal marker vimentin. To confirm the existence of EMT in ESCC, IHC was used to detect protein expression levels of epithelial marker E-cadherin and mesenchymal marker vimentin in ESCC tissues and corresponding normal mucosa. Cytoplasmic or membranal E-cadherin immunoreactivity (Fig. 1A and B) was examined in $40.00 \%$ of ESCC tissues, significantly lower than that in corresponding normal mucosa tissues $(76.84 \%$; $\mathrm{P}<0.01)$ (Table I). However, cytoplasmic vimentin immunoreactivity (Fig. 1C and D) was examined in $58.95 \%$ of ESCC tissues, significantly higher than that in corresponding normal mucosa tissues $(29.47 \%$; $\mathrm{P}<0.01$ ) (Table I). When we assessed the correlation of E-cadherin with vimentin protein in ESCC, a significantly negative correlation was found $(\mathrm{P}<0.01)$ (Table II). In addition, in ESCC tissues, E-cadherin expression was negatively correlated with histological grade $(\mathrm{P}<0.05)$, invasion depth $(\mathrm{P}<0.01)$ and lymph node metastasis $(\mathrm{P}<0.05)$ (Table $\mathrm{I})$, whereas vimentin protein expression was positively correlated with invasion depth $(\mathrm{P}<0.01)$ (Table I). These data demonstrated most ESCC tissues undergo EMT and acquire enhanced ability of invasiveness and metastasis.

The PTEN/PI3K signaling pathway is active and correlates with EMT and tumor progression in ESCC tissues. To investigate the role of the PTEN/PI3K pathway in ESCC, we examined the protein levels of PTEN and PI3K in ESCC tissues in comparison with the corresponding normal esophageal mucosa tissues by IHC. Cytoplasmic PTEN immunoreactivity (Fig. 1E and F) was examined in $49.47 \%$ of ESCC tissues, significantly lower than that in corresponding normal mucosa tissues $(89.47 \%$; $\mathrm{P}<0.01)$ (Table I). Moreover, cytoplasmic PI3K immunoreactivity (Fig. $1 \mathrm{G}$ and $\mathrm{H}$ ) was examined in $70.53 \%$ of ESCC tissues, significantly higher than that in corresponding normal mucosa tissues $(47.37 \%$; $\mathrm{P}<0.01$ ) (Table I). Furthermore, a significantly negative correlation was found between the expression of PTEN and PI3K
Table IV. Correlation of PTEN with E-cadherin and vimentin protein expression in ESCC tissues.

\begin{tabular}{|c|c|c|c|c|c|c|c|c|}
\hline \multirow[b]{2}{*}{ PTEN } & \multicolumn{4}{|c|}{ E-cadherin } & \multicolumn{4}{|c|}{ Vimentin } \\
\hline & + & - & $\mathrm{r}$ & P-value & + & - & $\mathrm{r}$ & P-value \\
\hline+ & 30 & 17 & 0.481 & 0.000 & 14 & 33 & -0.587 & 0.000 \\
\hline- & 8 & 40 & & & 42 & 6 & & \\
\hline
\end{tabular}

Table V. Correlation of PI3K with E-cadherin and vimentin protein expression in ESCC tissues.

\begin{tabular}{|c|c|c|c|c|c|c|c|c|}
\hline \multirow[b]{2}{*}{ PI3K } & \multicolumn{4}{|c|}{ E-cadherin } & \multicolumn{4}{|c|}{ Vimentin } \\
\hline & + & - & $\mathrm{r}$ & P-value & + & - & $\mathrm{r}$ & P-value \\
\hline+ & 19 & 48 & -0.368 & 0.000 & 45 & 22 & 0.258 & 0.012 \\
\hline- & 19 & 9 & & & 11 & 17 & & \\
\hline
\end{tabular}

protein in ESCC $(\mathrm{P}<0.01)$ (Table III). These data showed the PTEN/PI3K pathway is active in most ESCC tissues.

We also examined the correlation of PTEN and PI3K protein expression with $\mathrm{E}$-cadherin protein, vimentin protein and clinicopathological features in ESCC. As would be expected from the role of PTEN/PI3K signaling pathway in regulating EMT in ESCC, PTEN expression in ESCC had a positive association with E-cadherin and a negative association with vimentin $(\mathrm{P}<0.01)$ (Table IV), whereas PI3K expression presented contrary results $(\mathrm{P}<0.05)$ (Table V). Furthermore, as shown in Table I, a negative correlation was found between PTEN protein expression and histological grade $(\mathrm{P}<0.01)$, invasion depth $(\mathrm{P}<0.01)$ and lymph node metastasis $(\mathrm{P}<0.01)$. On the contrary, a positive correlation was found between PI3K protein expression and invasion depth $(\mathrm{P}<0.01)$ and lymph node metastasis $(\mathrm{P}<0.05)$. These data demonstrated that the PTEN/PI3K pathway plays a pivotal role in the process of EMT and tumor progression in ESCC. Additionally, our previous study also showed that TGF- $\beta 1$ correlates with EMT and tumor progression in ESCC tissues, suggesting TGF- $\beta 1$ and PTEN/PI3K signaling pathway fulfill overlapping roles and there may be a crosstalk between them.

Treatment of EC-1 cells with TGF- $\beta 1$ induces morphological and biochemical alterations consistent with EMT and enhanced aberrant cell invasiveness and migration. TGF- $\beta 1$ is a known inducer of EMT for a variety of cancer cells (16-18). In the present study, EC-1 cells belonging to human ESCC cell line were tested for their response to TGF- $\beta 1$. After treatment with $5 \mathrm{ng} / \mathrm{ml}$ of TGF- $\beta 1$, cell morphology was observed under a reversed light microscope at different time intervals (0, 24 and $48 \mathrm{~h})$. Compared to the untreated EC-1 cells (Fig. 2A), cells treated with TGF- $\beta 1$ were scattered, elongated and spindle-shaped, i.e., characteristic of fibroblasts (Fig. 2B and C). Notably, this morphological alteration was 

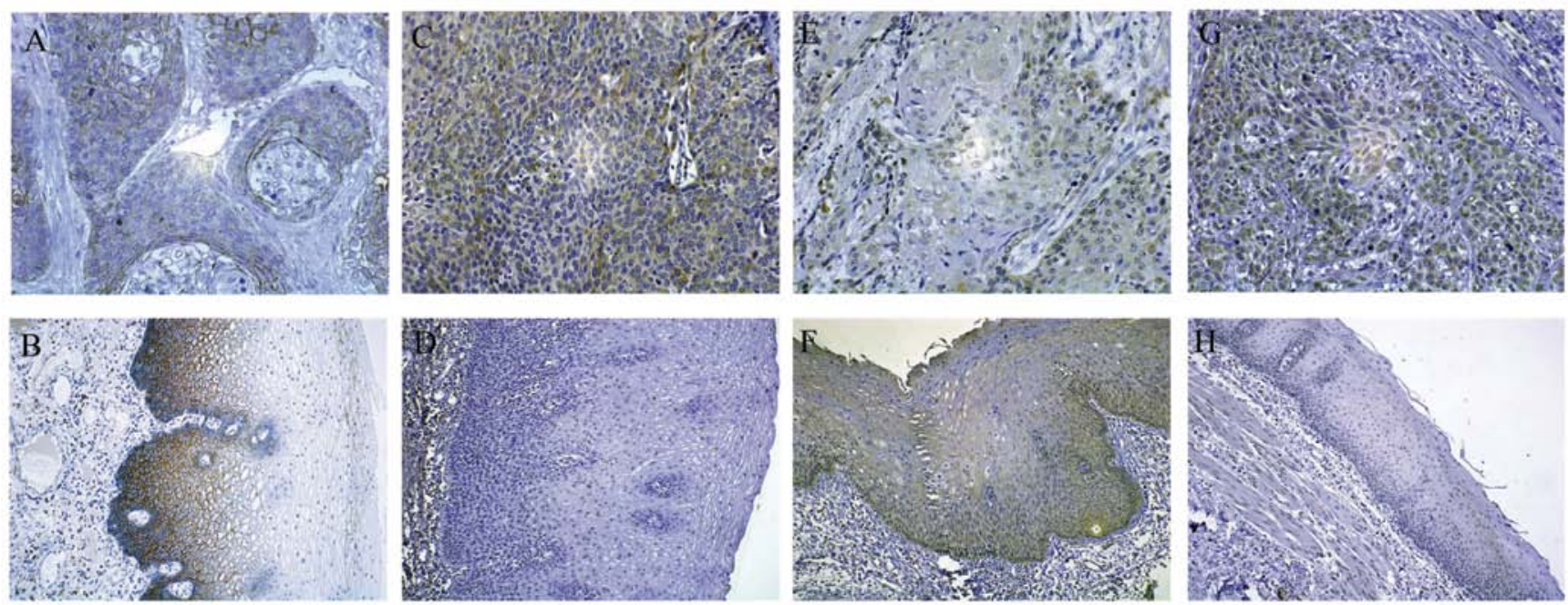

Figure 1. Immunohistochemical images of E-cadherin, vimentin, PTEN and PI3K proteins in representative samples from ESCC tissues and corresponding normal esophageal mucosa. (A) Strong membranal and cytoplasmic expression of E-cadherin in ESCC. (B) Strong membranal expression of E-cadherin in normal esophageal mucosa. (C) Strong cytoplasmic expression of vimentin in ESCC. (D) Negative expression of vimentin in normal esophageal mucosa. (E) Moderate cytoplasmic expression of PTEN in ESCC. (F) Strong cytoplasmic expression of PTEN in normal esophageal mucosa. (G) Strong cytoplasmic expression of PI3K in ESCC. (H) Weak cytoplasmic expression of PI3K in normal esophageal mucosa (x400 magnification for A, C, E and G; $x 200$ magnification for B, D, F and H).
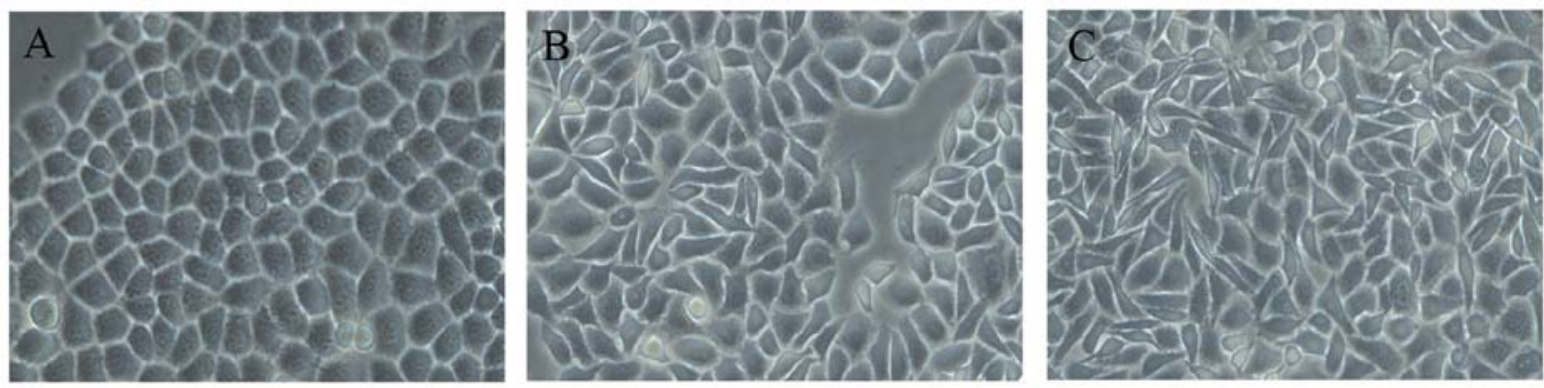

Figure 2. EC-1 cells grown to $70 \%$ confluency and then cultured in serum-free culture media overnight. Cells were treated with 5 ng/ml of TGF- $\beta 1$ for up to $48 \mathrm{~h}$ and cell morphology consistent with EMT was observed under a reversed light microscope. (A) Untreated EC-1 cells (treatment with $5 \mathrm{ng} / \mathrm{ml}$ of TGF- $\beta 1$ for $0 \mathrm{~h}$ ). (B) EC-1 cells treated with $5 \mathrm{ng} / \mathrm{ml}$ of TGF- $\beta 1$ for $24 \mathrm{~h}$. (C) EC-1 cells treated with $5 \mathrm{ng} / \mathrm{ml}$ of TGF- $\beta 1$ for $48 \mathrm{~h}$. All images were obtained at a x400 magnification.

time-dependent and occurred at $24 \mathrm{~h}$ (Fig. 2B), but became apparent at $48 \mathrm{~h}$ (Fig. 2C).

In line with the morphological alteration, EMT was characterized by suppression of epithelial marker E-cadherin and elevation of mesenchymal marker vimentin. Quantitative real-time PCR and western blot analysis showed that TGF- $\beta 1$ treatment induced a significant decrease in E-cadherin, but a significant increase in vimentin at both mRNA and protein levels in EC-1 cells as compared with those in untreated cells $(\mathrm{P}<0.01)$ (Fig. 3). Moreover, as shown in Fig. 3, treatment with TGF- $\beta 1$ displayed a significantly time-dependent change of E-cadherin and vimentin at mRNA and protein levels $(\mathrm{P}<0.05)$.

We next examined the effect of TGF- $\beta 1$ on cell invasiveness and migration by using invasion and scratch assay, respectively. The invasion assay demonstrated that EC-1 cells treated with $5 \mathrm{ng} / \mathrm{ml}$ of TGF- $\beta 1$ exhibited a significant increase in the number of cells that traversed the membrane toward a chemoattractant in a time-dependent manner as compared with untreated cells $(\mathrm{P}<0.01)$ (Fig. 4A). Additionally, the scratch assay showed that EC-1 cells treated with $5 \mathrm{ng} / \mathrm{ml}$ of TGF- $\beta 1$ resulted in a significant increase in the tumor cell migration distance as compared with untreated cells $(\mathrm{P}<0.01)$ (Fig. 4B and C).

Taken together, these data suggest that TGF- $\beta 1$ stimulation promotes EC-1 cells to undergo EMT and thus develop an aggressive and invasive phenotype.

TGF- $\beta 1$ stimulation activates PTEN/PI3K signaling and TGF- $\beta 1$-induced EMT is partly prevented by inhibition of the PTEN/PI3K signaling pathway in EC-1 cells. To analyze the crosstalk between TGF- $\beta 1$ and PTEN/PI3K signaling pathway during the process of EMT in ESCC cells, we first tested whether TGF- $\beta 1$ treatment activates the PTEN/PI3K pathway. As shown in Fig. 3, EC-1 cells treated with $5 \mathrm{ng} / \mathrm{ml}$ of TGF- $\beta 1$ for $48 \mathrm{~h}$ had lower PTEN but higher PI3K expression at both mRNA and protein levels as compared with the untreated cells $(\mathrm{P}<0.05)$, indicating TGF- $\beta 1$ treatment could activate the PTEN/PI3K signaling pathway.

Furthermore, we inhibited the PTEN/PI3K signaling pathway by pcDNA3.1-PTEN transfection for the purpose of demonstrating that TGF- $\beta 1$-induced EMT is driven by the 
$\mathbf{A}$

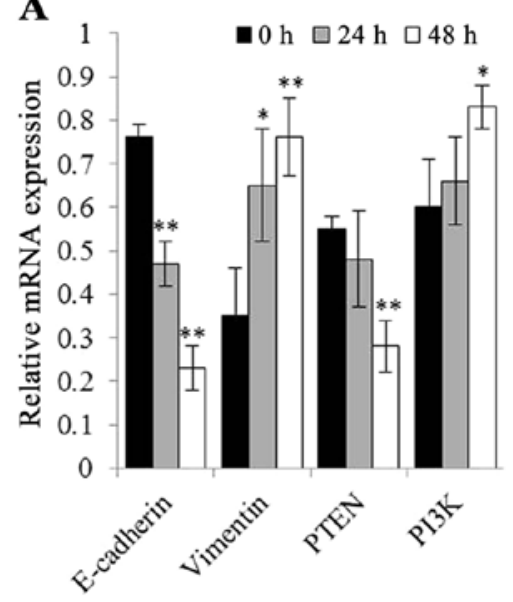

B

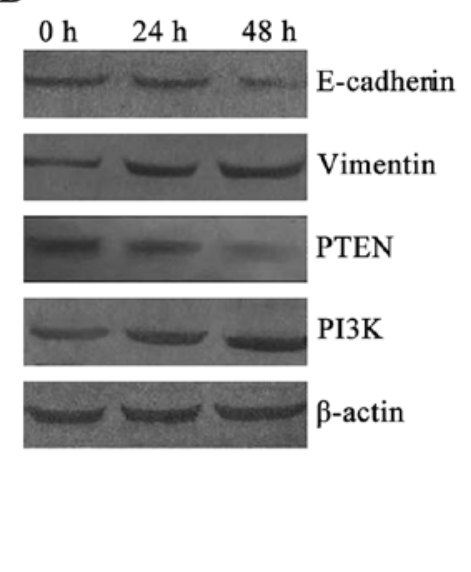

C

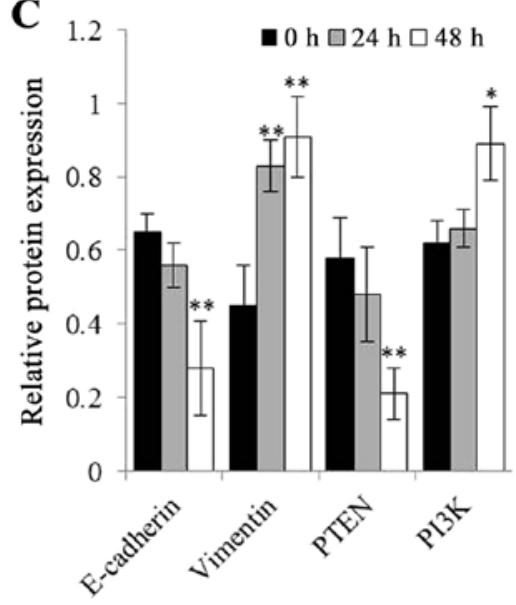

Figure 3. Quantitative real-time PCR and western blot analysis were used to detect mRNA and protein levels of E-cadherin, vimentin, PTEN and PI3K in EC-1 cells after treatment with $5 \mathrm{ng} / \mathrm{ml}$ of TGF- $\beta 1$ for different time intervals $(0,24$ and $48 \mathrm{~h})$, respectively. In EC- 1 cells, treatment with $5 \mathrm{ng} / \mathrm{ml}$ of TGF- $\beta 1$ reduced the epithelial marker E-cadherin but increased the mesenchymal marker vimentin mRNA and protein expression, which are the hallmark of EMT. Furthermore, this TGF- $\beta 1$-induced alteration of E-cadherin and vimentin occurred in a time-dependent manner. In addition, TGF- $\beta 1$ treatment decreased PTEN but increased PI3K mRNA and protein expression, leading to PTEN/PI3K signal activation. (A) mRNA levels of E-cadherin, vimentin, PTEN and PI3K in EC-1 cells treated with $5 \mathrm{ng} / \mathrm{ml}$ of TGF- $\beta 1$ for different time intervals $(0,24$ and $48 \mathrm{~h})$. (B and C) Protein levels of E-cadherin, vimentin, PTEN and PI3K in EC-1 cells treated with $5 \mathrm{ng} / \mathrm{ml}$ of TGF- $\beta 1$ for different time intervals $(0,24$ and $48 \mathrm{~h})$. $\beta$-actin was used as internal control. * $\mathrm{P}<0.05$ and ${ }^{* *} \mathrm{P}<0.01$, compared to the untreated EC-1 cells (treatment with $5 \mathrm{ng} / \mathrm{ml}$ of TGF- $\beta 1$ for $0 \mathrm{~h}$ ).

PTEN/PI3K signaling pathway. As shown in Fig. 5, after treatment with $5 \mathrm{ng} / \mathrm{ml}$ of TGF- $\beta 1$ for $48 \mathrm{~h}$, EC- 1 cells transfected with pcDNA3.1-PTEN had higher PTEN mRNA and protein expression as compared with cells untransfected or transfected with pcDNA3.1 empty vector $(\mathrm{P}<0.01)$, demonstrating the PTEN/PI3K pathway was inhibited by pcDNA3.1-PTEN directly. Moreover, accompanied by PTEN/PI3K signal inhibition, mRNA and protein levels of E-cadherin in EC-1 cells transfected with pcDNA3.1-PTEN were clearly higher than those in cells untransfected or transfected with pcDNA3.1 empty vector, whereas vimentin expression showed contrary results $(\mathrm{P}<0.01)$ (Fig. 5). These data suggested inhibition of the PTEN/PI3K signaling pathway by pcDNA3.1-PTEN prevents TGF- $\beta 1$-induced EMT to some extent.

The PTEN/PI3K pathway modulates TGF- $\beta 1$-induced tumor cell invasiveness and migration. To examine whether the PTEN/PI3K pathway also modulated TGF- $\beta 1$-induced tumor cell invasiveness and migration, we measured these two parameters after inhibition of the PTEN/PI3K signaling pathway by pcDNA3.1-PTEN in EC-1 cells. Invasion and scratch assay showed that, after treatment with $5 \mathrm{ng} / \mathrm{ml}$ of TGF- $\beta 1$ for 48 h, EC- 1 cells transfected with pcDNA3.1-PTEN exhibited a significant decrease in the number of traversed cells and the cell migration distance as compared with cells untransfected or transfected with pcDNA3.1 empty vector $(\mathrm{P}<0.01)$ (Fig. 4A-C). These data demonstrate that inhibition of the PTEN/PI3K signaling pathway by pcDNA3.1-PTEN counteracts TGF- $\beta 1$-induced tumor cell invasiveness and migration.

\section{Discussion}

Although numerous reports have implicated EMT in the progression of primary tumors towards invasion and metastasis
(8-10), the detailed molecular mechanisms and networks involved in TGF- $\beta$ and the PTEN/PI3K signaling pathway in EMT and progression of ESCC remain poorly understood.

EMT can be induced or regulated by various growth and differentiation factors including TGF- $\beta$, growth factors that act through receptor tyrosine kinases, such as FGF and HGF, and Notch and Wnt proteins (14). Among these, TGF- $\beta$ has received much attention as a major inducer of EMT during cancer progression (24). In the literature TGF- $\beta 1$ was shown to induce EMT in oral (16), liver (17) and pancreatic cancer (18). In the present study, we stimulated EC-1 cells with $5 \mathrm{ng} / \mathrm{ml}$ of TGF- $\beta 1$ for up to $48 \mathrm{~h}$ for the purpose of confirming TGF- $\beta 1$ induced EMT. The results showed that TGF- $\beta 1$ stimulation induced EC-1 cells to undergo a transition from the epithelial to the spindle-like mesenchymal morphology. This change was accompanied by the loss of E-cadherin and the gain of vimentin. In addition, the cells obtained increased ability of invasion and migration. Similar results were obtained for another ESCC cell line (EC9706) in a previous study (25). Thus, TGF- $\beta 1$ is also a key factor in EMT induction of ESCC cell lines.

Another notable factor involved in EMT is the PTEN/PI3K signaling pathway. The tumor suppressor gene PTEN is a dual protein/lipid phosphatase whose main substrate is PIP3, a product of PI3K (26). PTEN directly antagonizes PI3K function via abrogation of PIP3-mediated activation of downstream signaling events and ultimately participates in the regulation of the cell cycle, proliferation, apoptosis, cell adhesion and cancer progression $(6,19,27)$. In human tumors, PTEN activity is lost by mutation, deletion or promoter methylation silencing at high frequency $(26,28)$ and PI3K is constitutively activated in the loss of PTEN function $(29,30)$. Therefore, the PTEN/PI3K signaling pathway is frequently activated in a variety of human malignant tumors (31-33). Furthermore, activated PTEN/PI3K signaling pathway has also been identified as a central feature of EMT in tumor cells $(21,34)$. In 
A

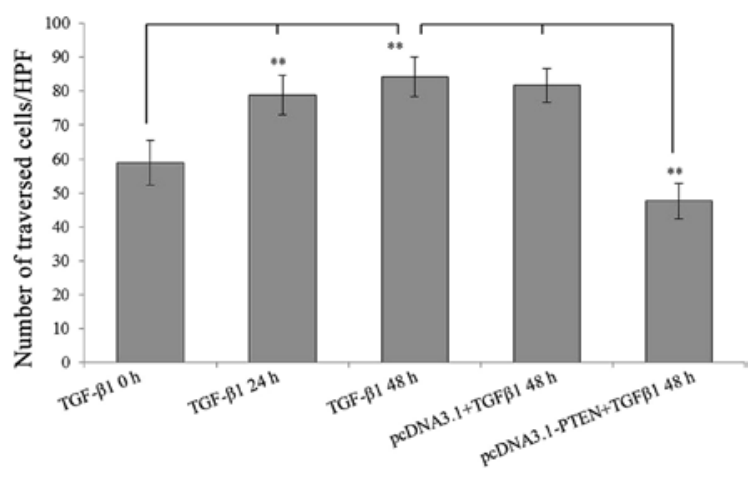

B
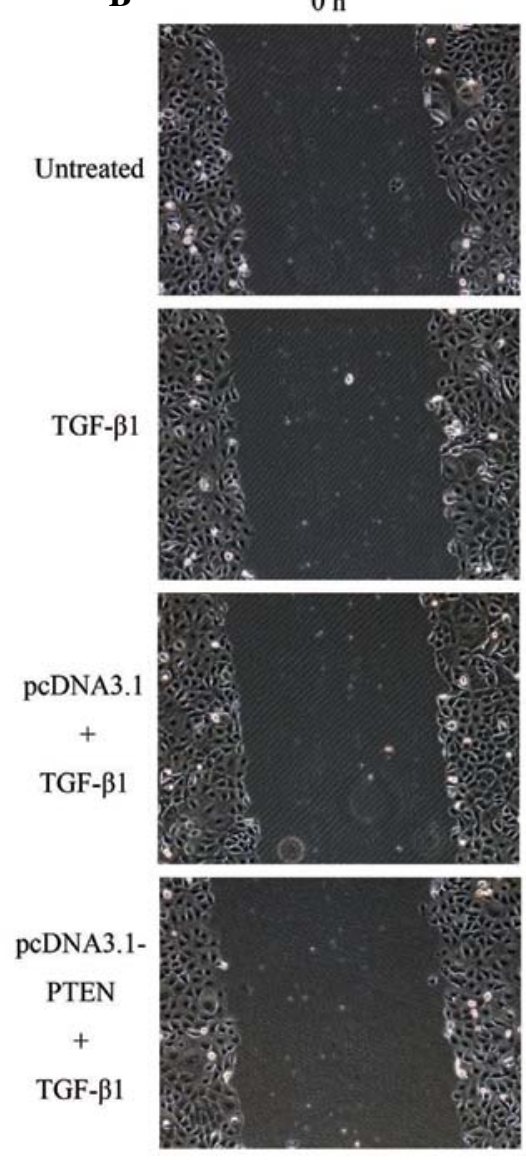

C

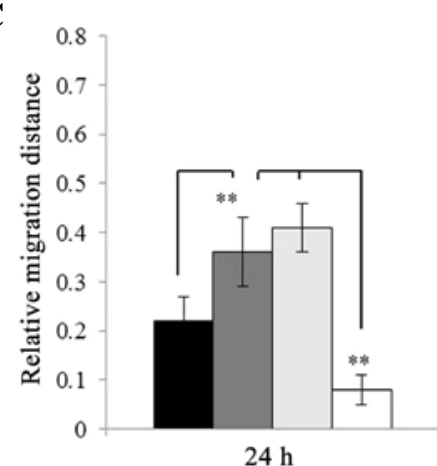

$24 \mathrm{~h}$
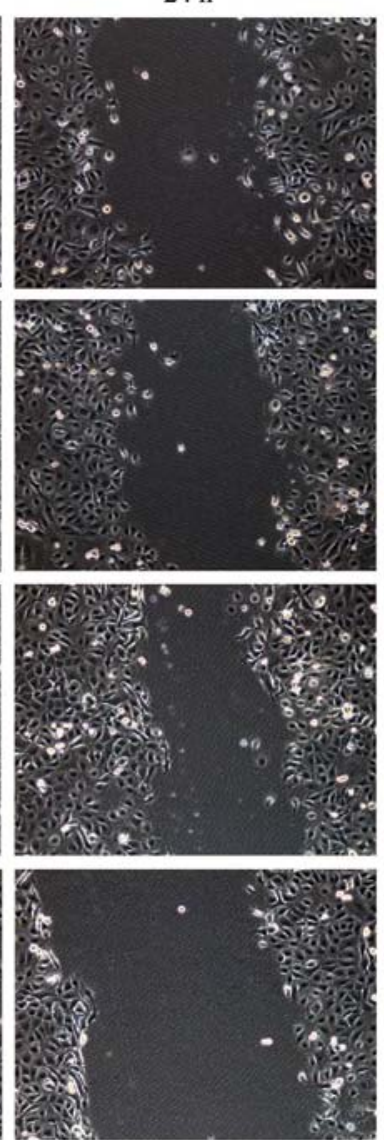

$48 \mathrm{~h}$
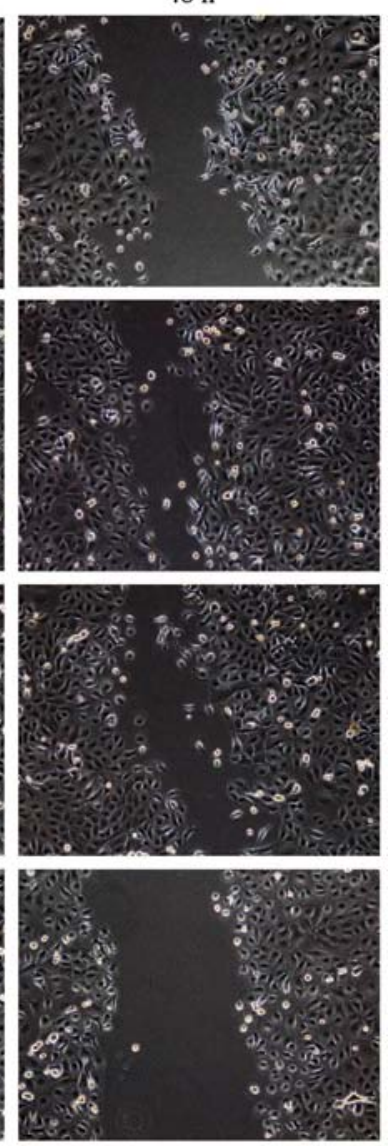

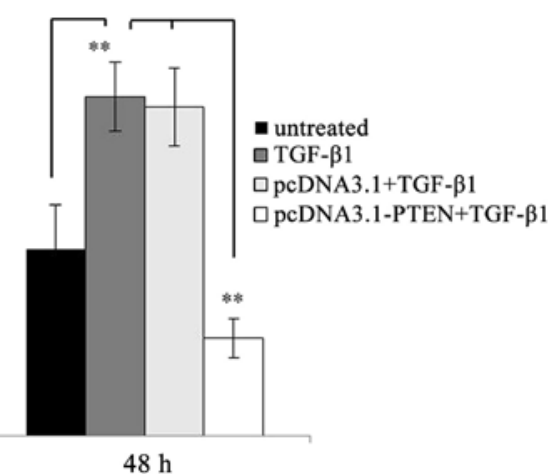

$48 \mathrm{~h}$

Figure 4. Invasiveness and migration of EC-1 cells are enhanced by TGF- $\beta 1$ stimulation, but are partly inhibited by pcDNA3.1-PTEN transfection. For the invasion assay, EC-1 cells untransfected and stably transfected with pcDNA3.1 or pcDNA3.1-PTEN were treated with $5 \mathrm{ng} / \mathrm{ml}$ of TGF- $\beta 1$ for different time intervals $(0,24$ and $48 \mathrm{~h})$, then seeded in the upper invasion chamber and incubated for an additional $24 \mathrm{~h}$, respectively. After incubation, cells that invaded through the Matrigel were fixed, stained and counted under a reversed light microscope at a x200 magnification. For the scratch assay, EC-1 cells untransfected and stably transfected with pcDNA3.1 or pcDNA3.1-PTEN were planted in 6-well plates, grown to $100 \%$ confluency and then scratched with a sterile $200 \mu 1$ pipette tip, respectively. After making the scratch, cells were treated with $5 \mathrm{ng} / \mathrm{ml}$ of TGF- $\beta 1$ for different time intervals $(0,24$ and $48 \mathrm{~h})$ and cell migration distances were quantitated by measuring the width of the wounds at a x 200 magnification under a reversed light microscope. (A) The number of traversed cells is presented as mean $\pm \mathrm{SD}$. (B) Images of EC-1 cell migration. (C) The relative migration distance is presented as 1 minus the average percent of wound closure by comparing with that at the zero time. ${ }^{* * *} \mathrm{P}<0.01$. 
A

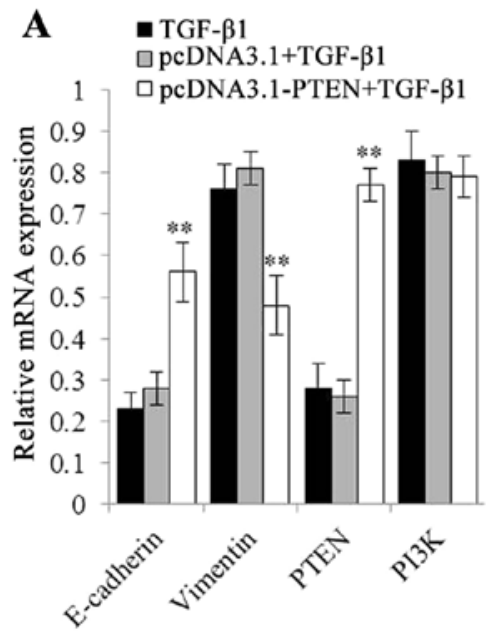

B

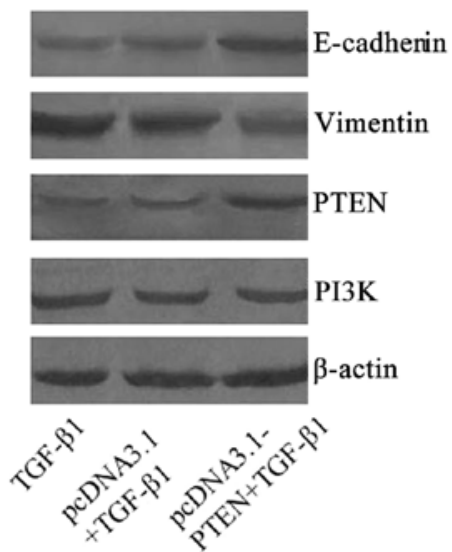

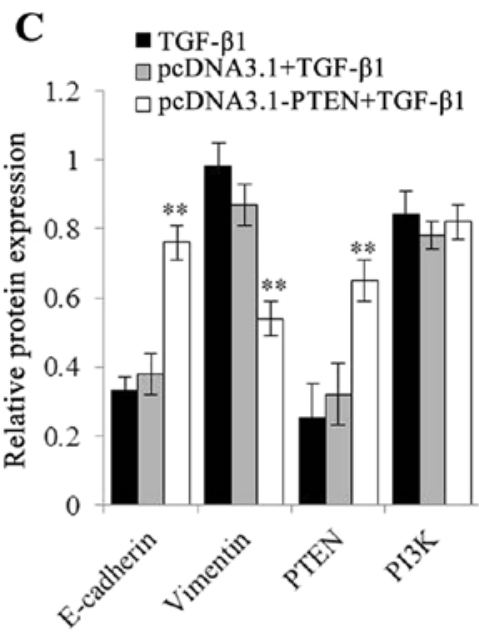

Figure 5. To demonstrate that TGF- $\beta 1$-induced EMT is driven by the PTEN/PI3K signaling pathway, EC-1 cells were stably transfected with pcDNA3.1-PTEN and subsequently treated with $5 \mathrm{ng} / \mathrm{ml}$ of TGF- $\beta 1$ for $48 \mathrm{~h}$. In EC-1 cells, inhibition of the PTEN/PI3K signaling pathway by pcDNA3.1-PTEN partly prevented TGF- $\beta 1$-induced EMT by increasing E-cadherin and decreasing vimentin expression at mRNA and protein levels. (A) mRNA levels of E-cadherin, vimentin, PTEN and PI3K in EC-1 cells untransfected and transfected with pcDNA3.1-PTEN or pcDNA3.1 empty vector. (B and C) Protein levels of E-cadherin, vimentin, PTEN and PI3K in EC-1 cells untransfected and transfected with pcDNA3.1-PTEN or pcDNA3.1 empty vector. $\beta$-actin was used as internal control. ${ }^{* *} \mathrm{P}<0.01$, compared to cells untransfected or transfected with pcDNA3.1 empty vector.

the present study, PTEN/PI3K signal was activated in ESCC and the activation of the PTEN/PI3K signaling pathway was negatively correlated with EMT marker E-cadherin, but was positively correlated with EMT marker vimentin and strongly contributed to tumor differentiation, invasion depth and lymph node in ESCC, suggesting the PTEN/PI3K signaling pathway plays a potential role in EMT and tumor progression. Indeed, in ESCC, in addition to the absence of functional PTEN, PI3K would be activated and would promote a series of EMT-inducing transcription factors, such as Snail (35). Upregulation of Snail, together with other transcription factors, leads to downregulation of EMT hallmark E-cadherin by inhibiting E-cadherin transcription $(36,37)$. Thus, ESCC cells undergo EMT and acquire enhanced invasive and migratory ability.

Although TGF- $\beta 1$ and PTEN/PI3K signaling pathway fulfill overlapping roles in EMT, a little known coordination mechanism has been proposed to explain their relationship in EMT to date. It has been reported that TGF- $\beta$ induces EMT by Smad-mediated transcription regulation $(11,14,15,38,39)$ and, moreover, TGF- $\beta /$ BMP-SMAD4 signaling pathway promotes prostate cancer EMT and progression by PTEN deletion (40). Furthermore, in mammary epithelial cells, TGF- $\beta$ can activate PI3K during EMT (41). Therefore, it is reasonable to postulate TGF- $\beta 1$-induced EMT is driven through the PTEN/PI3K signaling pathway. In the present study, we first hypothesized that the PTEN/PI3K signaling pathway is a downstream target of TGF- $\beta 1$ signal and examined PTEN and PI3K expression levels in EC- 1 cells after treatment with TGF- $\beta 1$. Western blot analysis and qPCR showed the protein and mRNA levels of PTEN were decreased, but PI3K was increased, indicating that downregulation of PTEN and upregulation of PI3K is influenced by TGF- $\beta 1$ stimulation (42). Although TGF- $\beta 1$ also leads to the downregulation of E-cadherin and upregulation of vimentin in EC-1 cells according to our previous results, it is unclear if this observed dysregulation of PTEN/PI3K and
E-cadherin/vimentin through TGF- $\beta 1$ stimulation is caused by a parallel mechanism or by a serial mechanism. Herein, we next inhibited PTEN/PI3K signaling pathway by pc-DNA3.1-PTEN application to confirm TGF- $\beta 1$-induced EMT via PTEN/PI3K pathway. As expected, our data showed that the enhancement of PTEN level by pc-DNA3.1-PTEN in TGF- $\beta 1$-treated EC-1 cells upregulated TGF- $\beta 1$-inhibited E-cadherin expression, but downregulated TGF- $\beta 1$-promoted vimentin expression, affirming the hypothesis that TGF- $\beta 1$-induced EMT is driven by the PTEN/PI3K signaling pathway. Additional evidence for the modulation of TGF- $\beta 1$-induced tumor cell invasiveness and migration by PTEN/PI3K signaling pathway comes from the invasion and scratch assay in vitro, which showed the inhibition of the PTEN/PI3K signaling pathway by pcDNA3.1-PTEN counteracts TGF- $\beta 1$-induced tumor cell invasiveness and migration. TGF- $\beta 1$ signal can regulate the cellular process by binding and phosphorylating TGF- $\beta$ receptors type I (T $\beta R$ I) and type II (T $\beta$ R II) (43), the activated T $\beta$ R I and T $\beta$ R II then bind to PI3K regulatory subunit $\mathrm{p} 85$, resulting in PI3K activation (44). In the presence of PTEN, activated PI3K function was antagonized and therefore TGF- $\beta 1$-induced EMT and EMT-associated mobility and invasiveness would be inhibited.

In conclusion, the present study suggests that TGF- $\beta 1$ and PTEN/PI3K signaling pathway contribute to EMT, and the PTEN/PI3K signaling pathway is a key regulator of TGF- $\beta 1$ induced EMT in ESCC. Disruption of the PTEN/PI3K pathway involved in TGF- $\beta 1$-induced EMT may provide possible routes for therapeutic intervention of ESCC.

\section{Acknowledgements}

This study was supported by grants from the National Natural Science Foundation of China (grant no. 81071970) and the Medical Science and Technology Program of Henan Province (grant no. 112102310195). We thank Professor Shihua Cao (the University of Hong Kong) for providing the EC-1 cell line. 


\section{References}

1. Pennathur A, Gibson MK, Jobe BA and Luketich JD: Oesophageal carcinoma. Lancet 381: 400-412, 2013.

2. Parkin DM, Bray F, Ferlay J and Pisani P: Global cancer statistics, 2002. CA Cancer J Clin 55: 74-108, 2005.

3. Ribeiro U Jr, Posner MC, Safatle-Ribeiro AV and Reynolds JC: Risk factors for squamous cell carcinoma of the oesophagus. $\mathrm{Br}$ J Surg 83: 1174-1185, 1996

4. Lu JC, Tao H, Zhang YQ, Zha WW, Qian PD, Li F and Xu KX: Extent of prophylactic postoperative radiotherapy after radical surgery of thoracic esophageal squamous cell carcinoma. Dis Esophagus 21: 502-507, 2008.

5. Ismail NI, Kaur G, Hashim H and Hassan MS: S100A4 overexpression proves to be independent marker for breast cancer progression. Cancer Cell Int 8: 12, 2008.

6. Wang H, Quah SY, Dong JM, Manser E, Tang JP and Zeng Q: PRL-3 down-regulates PTEN expression and signals through PI3K to promote epithelial-mesenchymal transition. Cancer Res 67: 2922-2926, 2007.

7. Thiery JP, Acloque H, Huang RY and Nieto MA: Epithelialmesenchymal transitions in development and disease. Cell 139: 871-890, 2009

8. Birchmeier C, Birchmeier W and Brand-Saberi B: Epithelialmesenchymal transitions in cancer progression. Acta Anat (Basel) 156: 217-226, 1996.

9. Hay ED: An overview of epithelio-mesenchymal transformation. Acta Anat (Basel) 154: 8-20, 1995.

10. Huber MA, Kraut N and Beug H: Molecular requirements for epithelial-mesenchymal transition during tumor progression. Curr Opin Cell Biol 17: 548-558, 2005.

11. Xu J, Lamouille S and Derynck R: TGF- $\beta$-induced epithelial to mesenchymal transition. Cell Res 19: 156-172, 2009.

12. Boyer B, Valles AM and Edme N: Induction and regulation of epithelial-mesenchymal transitions. Biochem Pharmacol 60: 1091-1099, 2000

13. Lee JM, Dedhar S, Kalluri R and Thompson EW: The epithelialmesenchymal transition: new insights in signaling, development, and disease. J Cell Biol 172: 973-981, 2006.

14. Moustakas A and Heldin $\mathrm{CH}$ : Signaling networks guiding epithelial-mesenchymal transitions during embryogenesis and cancer progression. Cancer Sci 98: 1512-1520, 2007.

15. Zavadil $J$ and Bottinger EP: TGF- $\beta$ and epithelial-to-mesenchymal transitions. Oncogene 24: 5764-5774, 2005.

16. Quan J, Elhousiny M, Johnson NW and Gao J: Transforming growth factor- $\beta 1$ treatment of oral cancer induces epithelialmesenchymal transition and promotes bone invasion via enhanced activity of osteoclasts. Clin Exp Metastasis 30: 659-670, 2013

17. Reichl P, Haider C, Grubinger M and Mikulits W: TGF- $\beta$ in epithelial to mesenchymal transition and metastasis of liver carcinoma. Curr Pharm Des 18: 4135-4147, 2012.

18. Ellenrieder V, Hendler SF, Boeck W, Seufferlein T, Menke A, Ruhland C, Adler G and Gress TM: Transforming growth factor $\beta 1$ treatment leads to an epithelial-mesenchymal transdifferentiation of pancreatic cancer cells requiring extracellular signal-regulated kinase 2 activation. Cancer Res 61: 4222-4228, 2001.

19. Zhang S and Yu D: PI(3)king apart PTEN's role in cancer. Clin Cancer Res 16: 4325-4330, 2010.

20. Cantley LC and Neel BG: New insights into tumor suppression: PTEN suppresses tumor formation by restraining the phosphoinositide 3-kinase/AKT pathway. Proc Natl Acad Sci USA 96: 4240-4245, 1999.

21. Wallin JJ, Guan J, Edgar KA, Zhou W, Francis R, Torres AC Haverty PM, Eastham-Anderson J, Arena S, Bardelli A, et al: Active PI3K pathway causes an invasive phenotype which can be reversed or promoted by blocking the pathway at divergent nodes. PLoS One 7: e36402, 2012.

22. Karamitopoulou E: Tumor budding cells, cancer stem cells and epithelial-mesenchymal transition-type cells in pancreatic cancer. Front Oncol 2: 209, 2013.

23. Zhang HY, Zheng XZ, Wang XH, Xuan XY, Wang F and Li SS S100A4 mediated cell invasion and metastasis of esophageal squamous cell carcinoma via the regulation of MMP-2 and E-cadherin activity. Mol Biol Rep 39: 199-208, 2012.
24. Wendt MK, Allington TM and Schiemann WP: Mechanisms of the epithelial-mesenchymal transition by TGF- $\beta$. Future Oncol 5: 1145-1168, 2009.

25. Sun Y, Li SS, Wang XH, Wang XJ and Yan AH: Transforming growth factor beta1 regulation of epithelial-mesenchymal transition in esophagus squamous cell carcinoma. Zhonghua Bing Li Xue Za Zhi 37: 542-548, 2008 (In Chinese).

26. Carnero A, Blanco-Aparicio C, Renner O, Link W and Leal JF: The PTEN/PI3K/AKT signalling pathway in cancer, therapeutic implications. Curr Cancer Drug Targets 8: 187-198, 2008.

27. Leslie NR, Yang X, Downes CP and Weijer CJ: PtdIns(3,4,5) $\mathrm{P}(3)$-dependent and -independent roles for PTEN in the control of cell migration. Curr Biol 17: 115-125, 2007.

28. Inoue K, Fry EA and Taneja P: Recent progress in mouse models for tumor suppressor genes and its implications in human cancer. Clin Med Insights Oncol 7: 103-122, 2013.

29. Simpson L and Parsons R: PTEN: life as a tumor suppressor. Exp Cell Res 264: 29-41, 2001.

30. Cully M, You H, Levine AJ and Mak TW: Beyond PTEN mutations: the PI3K pathway as an integrator of multiple inputs during tumorigenesis. Nat Rev Cancer 6: 184-192, 2006.

31. Li Y, Gao L, Luo X, Wang L, Gao X, Wang W, Sun J, Dou L, Li J, $\mathrm{Xu}$ C, et al: Epigenetic silencing of microRNA-193a contributes to leukemogenesis in $\mathrm{t}(8 ; 21)$ acute myeloid leukemia by activating the PTEN/PI3K signal pathway. Blood 121: 499-509, 2013.

32. Mulholland DJ, Kobayashi N, Ruscetti M, Zhi A, Tran LM, Huang J, Gleave M and Wu H: Pten loss and RAS/MAPK activation cooperate to promote EMT and metastasis initiated from prostate cancer stem/progenitor cells. Cancer Res 72: 1878-1889, 2012.

33. Bian Y, Hall B, Sun ZJ, Molinolo A, Chen W, Gutkind JS, Waes CV and Kulkarni AB: Loss of TGF- $\beta$ signaling and PTEN promotes head and neck squamous cell carcinoma through cellular senescence evasion and cancer-related inflammation. Oncogene 31: 3322-3332, 2012.

34. Larue L and Bellacosa A: Epithelial-mesenchymal transition in development and cancer: role of phosphatidylinositol 3 kinase/AKT pathways. Oncogene 24: 7443-7454, 2005.

35. Chen KC, Chen CY, Lin CJ, Yang TY, Chen TH, Wu LC and Wu CC: Luteolin attenuates TGF- $\beta 1$-induced epithelialmesenchymal transition of lung cancer cells by interfering in the PI3K/Akt-NF-кB-Snail pathway. Life Sci 93: 924-933, 2013.

36. Barrallo-Gimeno A and Nieto MA: The Snail genes as inducers of cell movement and survival: implications in development and cancer. Development 132: 3151-3161, 2005.

37. Cano A, Perez-Moreno MA, Rodrigo I, Locascio A, Blanco MJ, del Barrio MG, Portillo F and Nieto MA: The transcription factor snail controls epithelial-mesenchymal transitions by repressing E-cadherin expression. Nat Cell Biol 2: 76-83, 2000.

38. Moustakas A and Heldin CH: Induction of epithelial-mesenchymal transition by transforming growth factor $\beta$. Semin Cancer Biol 22: 446-454, 2012.

39. Lv ZD, Kong B, Li JG, Qu HL, Wang XG, Cao WH, Liu XY, Wang Y, Yang ZC, Xu HM and Wang HB: Transforming growth factor- $\beta 1$ enhances the invasiveness of breast cancer cells by inducing a Smad2-dependent epithelial-to-mesenchymal transition. Oncol Rep 29: 219-225, 2013.

40. Ju X, Casimiro MC, Gormley M, Meng H, Jiao X, Katiyar S, Crosariol M, Chen K, Wang M, Quong AA, et al: Identification of a cyclin D1 network in prostate cancer that antagonizes epithelial-mesenchymal restraint. Cancer Res 74: 508-519, 2014.

41. Bakin AV, Tomlinson AK, Bhowmick NA, Moses HL and Arteaga CL: Phosphatidylinositol 3-kinase function is required for transforming growth factor $\beta$-mediated epithelial to mesenchymal transition and cell migration. J Biol Chem 275: 36803-36810, 2000.

42. Li DM and Sun H: TEP1, encoded by a candidate tumor suppressor locus, is a novel protein tyrosine phosphatase regulated by transforming growth factor $\beta$. Cancer Res 57: 2124-2129, 1997.

43. Blobe GC, Schiemann WP and Lodish HF: Role of transforming growth factor $\beta$ in human disease. N Engl J Med 342: 1350-1358, 2000.

44. Krymskaya VP, Hoffman R, Eszterhas A, Ciocca V and Panettieri RA Jr: TGF- $\beta 1$ modulates EGF-stimulated phosphatidylinositol 3-kinase activity in human airway smooth muscle cells. Am J Physiol 273: L1220-L1227, 1997. 